Pacific Journal of Mathematics

ON DIRECT SUMS AND PRODUCTS OF MODULES 


\title{
ON DIRECT SUMS AND PRODUCTS OF MODULES
}

\author{
Stephen U. Chase
}

A well-known theorem of the theory of abelian groups states that the direct product of an infinite number of infinite cyclic groups is not free ([6], p. 48.) Two generalizations of this result to modules over various rings have been presented in earlier papers of the author ([3], [4].) In this note we exhibit a broader generalization which contains the preceding ones as special cases.

Moreover, it has other applications. For example, it yields an easy proof of a part of a result of Baumslag and Blackburn [2] which gives necessary conditions under which the direct sum of a sequence of abelian groups is a direct summand of their direct product. We also use it to prove the following variant of a result of Baer [1]: If a torsion group $T$ is an epimorphic image of a direct product of a sequence of finitely generated abelian groups, then $T$ is the direct sum of a divisible group and a group of bounded order. Finally, we derive a property of modules over a Dedekind ring which, for the ring $Z$ of rational integers, reduces to the following recent theorem of Rotman [10] and Nunke [9]: If $A$ is an abelian group such that $\operatorname{Ext}_{z}(A, T)=0$ for any torsion group $T$, then $A$ is slender.

In this note all rings have identities and all modules are unitary.

1. The main theorem. Our discussion will be based on the following technical device.

DEFINITION 1.1. Let $\mathscr{F}$ be a collection of principal right ideals of a ring $R$. $\mathscr{F}$ will be called a filter of principal right ideals if, whenever $a R$ and $b R$ are in $\mathscr{F}$, there exists $c \in a R \cap b R$ such that $c R$ is in $\mathscr{F}$.

We proceed immediately to the principal result of this note.

THEOREM 1.2. Let $A^{(1)}, A^{(2)}, \cdots$ be a sequence of left modules over $a$ ring $R$, and set $A=\prod_{i=1}^{\infty} A^{(i)}, A_{n}=\prod_{i=n+1}^{\infty} A^{(i)}$. Let $C=\sum_{\alpha} \oplus C_{\alpha}$, where $\left\{C_{\alpha}\right\}$ is a family of left $R$-modules and $\alpha$ traces an index set $I$. Let $f: A \rightarrow C$ be an $R$-homomorphism, and denote by $f_{\alpha}: A \rightarrow C_{a}$ the composition of $f$ with the projection of $C$ onto $C_{\alpha}$. Finally, let $\mathscr{F}$ be a filter of principal right ideals of $R$. Then there exists aR in $\mathscr{F}$ and an integer $n>0$ such that $f_{a}\left(a A_{n}\right) \cong \bigcap_{b \hat{R} \in \mathscr{F}} b C_{a}$ for all but $a$ finite number of $\alpha$ in $I$.

Proof. Assume that the statement is false. We shall first construct 
inductively sequences $\left\{x_{n}\right\} \subseteq A,\left\{a_{n} R\right\} \subseteq \mathscr{F}$, and $\left\{\alpha_{n}\right\} \subseteq I$ such that the following conditions hold:
( i ) $\quad a_{n} R \supseteqq a_{n+1} R$.
(ii) $x_{n} \in a_{n} A_{n}$.
(iii) $f_{a_{n}}\left(x_{n}\right) \not \equiv 0\left(\bmod a_{n+1} C_{a_{n}}\right)$.
(iv) $f_{\alpha_{n}}\left(x_{k}\right)=0$ for $k<n$.

We proceed as follows. Select any $a_{1} R$ in $\mathscr{F}$. Then there exists $\alpha_{1} \in I$ such that $f_{\alpha_{1}}\left(a_{1} A_{1}\right) \not \subset \bigcap_{b R \in \mathscr{F}} b C_{\alpha_{1}}$, and hence we may select $b R$ in $\mathscr{F}$ such that $f_{\alpha_{1}}\left(a_{1} A_{1}\right) \not \subset b C_{\alpha_{1}}$. Since $\mathscr{F}$ is a filter of principal right ideals, there exists $a_{2} \in a_{1} R \cap b R$ such that $a_{2} R \in \mathscr{F}$, in which case $f_{\alpha_{1}}\left(a_{1} A_{1}\right) \not \subset a_{2} C_{\alpha_{1}}$. Hence there exists $x_{1} \in a_{1} A_{1}$ such that $f_{\alpha_{1}}\left(x_{1}\right) \not \equiv 0$ $\left(\bmod a_{2} C_{\alpha_{1}}\right)$. Then conditions (i)-(iv) above are satisfied for $n=1$.

Proceed by induction on $n$; assume that the sequences $\left\{x_{k}\right\}$ and $\left\{\alpha_{k}\right\}$ have been constructed for $k<n$ and the sequence $\left\{a_{k} R\right\}$ has been constructed for $k \leqq n$ such that conditions (i)-(iv) are satisfied. Now, there exist $\beta_{1}, \cdots, \beta_{r} \in I$ such that, if $\alpha \neq \beta_{1}, \cdots, \beta_{r}$, then $f_{\alpha}\left(x_{k}\right)=0$ for all $k<n$. We may then select $\alpha_{n} \neq \beta_{1}, \cdots, \beta_{r}$ such that $f_{\alpha_{n}}\left(a_{n} A_{n}\right) \not \subset \bigcap_{b R \in \mathscr{F}} b C_{\alpha_{n}}$; for, if we could not do so, then the theorem would be true. Hence there exists $b R \in \mathscr{F}$ such that $f_{a_{n}}\left(a_{n} A_{n}\right) \not \subset b C_{a_{n}}$. Since $\mathscr{F}$ is a filter of principal right ideals, there exists $a_{n+1} \in a_{n} R \cap b R$ such that $a_{n+1} R$ is in $\mathscr{F}$, in which case $f_{\alpha_{n}}\left(a_{n} A_{n}\right) \not \subset a_{n+1} C_{\alpha_{n}}$. Thus we may select $x_{n} \in a_{n} A_{n}$ such that $f_{a_{n}}\left(x_{n}\right) \not \equiv 0\left(\bmod a_{n+1} C_{\alpha_{n}}\right)$. It is then clear that the sequences $\left\{x_{k}\right\}$ and $\left\{\alpha_{k}\right\}$ for $k \leqq n$ and $\left\{a_{k} R\right\}$ for $k \leqq n+1$ satisfy conditions (i)-(iv), and hence the construction of all three sequences is complete.

Now write $x_{k}=\left(x_{k}^{(i)}\right)$, where $x_{k}^{(i)} \in A^{(i)}$. Since $x_{k} \in a_{k} A_{k}, x_{k}^{(i)}=0$ for $k>i$, and $x^{(i)}=\sum_{k=1}^{\infty} x_{k}^{(i)}$ is a well-defined element of $A^{(i)}$. Also, since $a_{n} R \supseteqq a_{n+1} R \supseteqq \cdots$, it follows that there exists $y_{n}^{(i)} \in A^{(i)}$ such that $x^{(i)}=$ $x_{1}^{(i)}+\cdots+x_{n}^{(i)}+a_{n+1} y_{n}^{(i)}$. Therefore, setting $x=\left(x^{(i)}\right)$ and $y_{n}=\left(y_{n}^{(i)}\right)$, we see that $x=x_{1}+\cdots+x_{n}+a_{n+1} y_{n}$ for all $n \geqq 1$.

It follows immediately from inspection of conditions (iii) and (iv) above that $\alpha_{i} \neq \alpha_{j}$ if $i \neq j$. Hence there exists $n$ such that $f_{\alpha_{n}}(x)=0$. Writing $x=x_{1}+\cdots+x_{n}+a_{n+1} y_{n}$ as above, we may then apply $f_{\alpha_{n}}$ and use condition (iv) to conclude that $f_{a_{n}}\left(x_{n}\right)=-a_{n+1} f_{\alpha_{n}}\left(y_{n}\right) \equiv 0$ (mod $a_{n+1} C_{a_{n}}$ ), contradicting condition (iii). The proof of the theorem is hence complete.

In the following discussion we shall use the symbol $|X|$ to denote the cardinality of the set $X$.

CoRollary 1.3 ([3], Theorem 3.1, p. 464). Let $R$ be a ring, and $A=\prod_{\alpha \in J} R^{(\alpha)}$, where $R^{(\alpha)} \approx R$ as a left $R$-module and $|J| \geqq \aleph_{0}$. Suppose that $A$ is a pure submodule of $C=\sum_{\beta} \oplus C_{\beta}$, where each $C_{\beta}$ is a left $R$ - 
module and $\left|C_{\beta}\right| \leqq|J| .^{1}$ Then $R$ must satisfy the descending chain condition on principal right ideals.

Proof. Since $J$ is an infinite set, it is easy to see that $A \approx \prod_{i=1}^{\infty} A^{(i)}$, where $A^{(i)} \approx A$, and so without further ado we shall identify $A$ with $\prod_{i=1}^{\infty} A^{(i)}$. Let $f: A \rightarrow C$ be the inclusion mapping, and $f_{\beta}: A \rightarrow C_{\beta}$ be the composition of $f$ with the projection of $C$ onto $C_{\beta}$. Finally, set $A_{n}=\prod_{i=n+1}^{\infty} A^{(i)}$.

Suppose that the statement is false. Then there exists a strictly descending infinite chain $a_{1} R \supsetneqq a_{2} R \supsetneqq \cdots$ of principal right ideals of $R$. These ideals obviously constitute a filter of principal right ideals of $R$, and so we may apply Theorem 1.2 to conclude that there exists $n \geqq 1$ and $\beta_{1}, \cdots, \beta_{r}$ such that $f_{\beta}\left(a_{n} A_{n}\right) \subseteq a_{n+1} C_{\beta}$ for $\beta \neq \beta_{1}, \cdots, \beta_{r}$.

Now let $C^{\prime}=C_{\beta_{1}} \oplus \cdots \oplus C_{\beta_{r}}$; then the projection of $C$ onto $C^{\prime}$ induces a $Z$-homomorphism $g: a_{n} C / a_{n+1} C \rightarrow a_{n} C^{\prime} / a_{n+1} C^{\prime}$, where $Z$ is the ring of rational integers. Also, the restriction of $f$ to $A_{n}$ induces a $Z$ homomorphism $h: a_{n} A_{n} / a_{n+1} A_{n} \rightarrow a_{n} C / a_{n+1} C . \quad A_{n}$ is a direct summand of $A$, which is a pure submodule of $C$, and so $A_{n}$ is likewise a pure submodule of $C$. Hence $h$ is a monomorphism. We may then apply the conclusion of the preceding paragraph to obtain that the composition $g h$ is a monomorphism. In particular, $\left|a_{n} A_{n}\right| a_{n+1} A_{n}|\leqq| a_{n} C^{\prime}\left|a_{n+1} C^{\prime}\right| \leqq\left|C^{\prime}\right|$.

Observe that $\left|C^{\prime}\right| \leqq|J|$, since $J$ is infinite and $\left|C_{\beta}\right| \leqq|J|$ for all $\beta$. However, since $a_{n} R \neq a_{n+1} R, a_{n} R / a_{n+1} R$ contains at least two elements; therefore $\left|a_{n} A_{n} / a_{n+1} A_{n}\right|=\left|a_{n} A / a_{n+1} A\right| \geqq 2^{|J|}>|J|$. We have thus reached a contradiction, and the corollary is proved.

2. Applications to integral domains. Throughout this section $R$ will be an integral domain. If $C$ is an $R$-module, we shall denote the maximal divisible submodule of $C$ by $d(C)$. In addition, we shall write $R^{\omega} C=\bigcap a C$, where $a$ traces the nonzero elements of $R$.

Our principal result concerning modules over integral domains is the following theorem.

Theorem 2.1. Let $\left\{A^{(i)}\right\}$ be a sequence of $R$-modules, and set $A=$ $\prod_{i=1}^{\infty} A^{(i)}, A_{n}=\prod_{i=n+1}^{\infty} A^{(i)}$. Let $C=\sum_{\alpha} \oplus C_{a}$, where each $C_{a}$ is an $R$ module. Let $f: A \rightarrow C$ be an $R$-homomorphism, and $f_{a}: A \rightarrow C_{a}$ be the composition of $f$ with the projection of $C$ onto $C_{\alpha}$. Then there exists an integer $n \geqq 1$ and $a \in R, a \neq 0$, such that $a f_{\alpha}\left(A_{n}\right) \subseteq R^{\omega} C_{a}$ for all but finitely many $\alpha$.

Proof. Let $\mathscr{F}$ be the set of all nonzero principal ideals of $R$. Since $R$ is an integral domain, it is clear that $\mathscr{F}$ is a filter of principal ideals. The theorem then follows immediately from Theorem 1.2.

$1 \mathrm{~A}$ is a pure submodule of $C$ if $A \cap a C=a A$ for all $a \in R$. 
CoRollary 2.2 (see [4].) Same hypotheses and notation as in Theorem 2.1, with the exception that now each $C_{a}$ is assumed to be torsion-free. Then there exists an integer $n \geqq 1$ such that $f_{\alpha}\left(A_{n}\right) \subseteq d\left(C_{\alpha}\right)$ for all but finitely many $\alpha$. In particular, if each $C_{\alpha}$ is reduced (i.e., has no divisible submodules) then $f_{\alpha}\left(A_{n}\right)=0$ for all but finitely many $\alpha$.

Proof. This follows immediately from Theorem 2.1 and the trivial observation that, since each $C_{\alpha}$ is torsion-free, $R^{\omega} C_{\alpha}=d\left(C_{\alpha}\right)$.

Next we present our proof of the afore-mentioned result of Baumslag and Blackburn concerning direct summands of direct products of abelian groups ([2], Theorem 1, p. 403.)

THEOREM 2.3. Let $\left\{A^{(i)}\right\}$ be a sequence of modules over an integral domain $R$, and set $A=\prod_{i=1}^{\infty} A^{(i)}, C=\sum_{i=1}^{\infty} \oplus A^{(i)}$ (then $C$ is, in the usual way, a submodule of $A$.) If $C$ is a direct summand of $A$, then there exists $n \geqq 1$ and $a \neq 0$ in $R$ such that $a A^{(i)} \subseteq d\left(A^{(i)}\right)$ for $i>n$.

Proof. Assume that $C$ is a direct summand of $A$, and let $f: A \rightarrow C$ be the projection. Then the composition of $f$ with the projection of $C$ onto $A^{(i)}$ is an epimorphism $f_{i}: A \rightarrow A^{(i)}$. We then obtain from an easy application of Theorem 2.1 that there exists $n \geqq 1$ and $a \neq 0$ in $R$ such that $a f_{i}(A) \cong R^{\omega} A^{(i)}$. Since each $f_{i}$ is an epimorphism, it follows that $a A^{(i)} \subseteq R^{\omega} A^{(i)}$ for $i>n$.

Now let $z \in R^{\omega} A^{(i)}$, where $i>n$. If $b \neq 0$ is in $R$, then there exists $x \in A^{(i)}$ such that $a b x=z$. Hence, setting $y=a x$, we have that $y \in R^{\omega} A^{(i)}$ and $b y=z$. It then follows that $R^{\omega} A^{(i)}$ is divisible, and so $R^{\omega} A^{(i)} \subseteq$ $d\left(A^{(i)}\right)$. Therefore $a A^{(i)} \cong R^{\omega} A^{(i)} \subseteq d\left(A^{(i)}\right)$ for $i>n$, completing the proof of the theorem.

We end this section with a proposition which will be useful in the proof of some later results.

Proposition 2.4. Let $\left\{A^{(i)}\right\}$ be a sequence of finitely generated modules over an integral domain $R$, and set $A=\prod_{i=1}^{\infty} A^{(i)}$. Let $C=$ $\sum_{\alpha} \oplus C_{\alpha}$, where each $C_{\alpha}$ is a finitely generated torsion $R$-module. If $f: A \rightarrow C$ is an $R$-homomorphism, then there exists $c \in R$ such that $c f(A)=$ 0 but $c \neq 0$.

Proof. As before we let $\mathscr{F}$ be the filter of all nonzero principal ideals of $R$. Clearly $R^{\omega} C_{\alpha}=0$ for all $\alpha$, and so we may apply Theorem 2.1 to obtain $a \neq 0$ in $R$ and an integer $n>0$ such that $a f_{\alpha}\left(A_{n}\right)=0$ for all but finitely many $\alpha$, where $A_{n}=\prod_{i=n+1}^{\infty} A^{(i)}$ and $f_{\alpha}: A \rightarrow C_{\alpha}$ is defined as before. Say this condition holds for $\alpha \neq \alpha_{1}, \cdots, \alpha_{r}$; then, since each $C_{\alpha}$ is finitely generated and torsion, there exists $a^{\prime} \neq 0$ in $R$ such that $a^{\prime} C_{\alpha_{i}}=0$ for $i=1, \cdots, r$, in which case $a a^{\prime} f\left(A_{n}\right)=0$. Since 
each $A^{(i)}$ is finitely generated and $C$ is a torsion module, there exists $a^{\prime \prime} \neq 0$ in $R$ such that $a^{\prime \prime} f\left(A^{(i)}\right)=0$ for $i \leqq n$. Set $c=a a^{\prime} a^{\prime \prime}$; then $c \neq 0$ and, since $A=A^{(1)} \oplus \cdots \oplus A^{(n)} \oplus A_{n}$, it is clear that $\operatorname{cf}(A)=0$, completing the proof of the proposition.

3. Applications to Abelian groups. This section is devoted to a discussion of the results of Baer, Rotman, and Nunke mentioned in the introduction.

Theorem 3.1 (see [1], Lemma 4.1, p. 231). Let $\left\{A^{(i)}\right\}$ be a sequence of finitely generated modules over a principal ideal domain $R$, and set $A=\prod_{i=1}^{\infty} A^{(i)}$. If $C$ is a torsion $R$-module which is an epimorphic image of $A$, then $C$ is the direct sum of a divisible module and a module of bounded order.

Proof. For each prime $p$ in $R$, let $C_{p}$ be the $p$-primary component of $C$ and $C_{p}^{\prime}$ be a basic submodule of $C_{p}$ (see [5], p. 98;) i.e., $C_{p}^{\prime}$ is a direct sum of cyclic modules and is a pure submodule of $C_{p}$, and $C_{p} / C_{p}^{\prime}$ is divisible. ${ }^{2}$ Set $C^{\prime}=\sum_{p} \oplus C_{p}^{\prime}$; then, since $C=\sum_{p} \oplus C_{p}, C^{\prime}$ is a pure submodule of $C$ and $C / C^{\prime}$ is divisible. Also, $C^{\prime}$ is a direct sum of cyclic modules.

We now apply the fundamental result of Szele ([5], Theorem 32.1, p. 106) to conclude that $C_{p}^{\prime}$ is an endomorphic image of $C_{p}$ for each prime $p$, from which it follows that $C^{\prime}$ is an endomorphic image of $C$. Since by hypothesis $C$ is an epimorphic image of $A$, we then see that there exists an epimorphism $f: A \rightarrow C^{\prime}$. By Proposition 2.4, there exists $c \neq 0$ in $R$ such that $c C=c f(A)=0$; i.e., $C^{\prime}$ has bounded order. Since $C^{\prime}$ is a pure submodule of $C$, we may apply Theorem 7 of [6] (p. 18) to conclude that $C^{\prime}$ is a direct summand of $C$. Since $C / C^{\prime}$ is divisible, the proof is complete.

For the case in which $R$ is the ring of rational integers, the assertion of Theorem 3.1 follows from the work of Nunke [9].

In the remainder of this note, $R$ will be a Dedekind ring which is not a field. If $A$ and $C$ are $R$-modules, we shall write $\operatorname{Ext}(A, C)$ for $\operatorname{Ext}_{R}^{1}(A, C)$. The following two lemmas are well-known, but to our knowledge have not appeared explicitly in the literature.

Lemma 3.2. Let $a \neq 0$ be a nonunit in $R$, and let $A$ and $C$ be $R$ modules. Assume that $a C=0$, and a operates faithfully on $A$ (i.e., $a x=0$ for $x \in A$ only if $x=0$.) Then $\operatorname{Ext}(A, C)=0$.

2 The definition and properties of basic submodules used here, as well as the theorem of Szele applied in the following paragraph, are in [5] given only for the special case in which $R$ is the ring of rational integers. However, it is well-known that these results can be trivially extended to modules over an arbitrary principal ideal domain. 
Proof. Since $a$ operates faithfully on $A$, we obtain the exact sequence-

$$
0 \longrightarrow A \stackrel{m_{a}}{\longrightarrow} A \longrightarrow A / a A \longrightarrow 0
$$

where $m_{a}$ is defined by $m_{a}(x)=a x$. This gives rise to the exact cohomology sequence-

$$
\operatorname{Ext}(A, C) \stackrel{m_{a}^{*}}{\longrightarrow} \operatorname{Ext}(A, C) \longrightarrow 0
$$

where $m_{a}^{*}(u)=a u$ for $u$ in $\operatorname{Ext}(A, C)$. But, since $a C=0$, we have that $m_{a}^{*}=0$, and so it follows from exactness that $\operatorname{Ext}(A, C)=0$, completing the proof.

LeMma 3.3. Let $a \neq 0$ be a nonunit in $R$, and $A, C$ be $R$-modules. Assume that a operates faithfully on $A$. Then the following statements are equivalent:

(a) a operates faithfully on $\operatorname{Ext}(A, C)$.

(b) The natural mapping $\operatorname{Hom}(A, C) \rightarrow \operatorname{Hom}(A, C / a C)$ is an epimorphism.

Proof. Consider the exact sequence-

$$
0 \longrightarrow C_{a} \longrightarrow C \stackrel{m_{a}}{\longrightarrow} C \longrightarrow C / a C \longrightarrow 0
$$

where $C_{a}=\{x \in C / a x=0\}$ and $m_{a}$ is defined as in Lemma 3.2. This sequence may be broken up into the following short exact sequences:

$$
\begin{aligned}
0 \longrightarrow C_{a} \longrightarrow C \stackrel{\mu}{\longrightarrow} a C \longrightarrow 0 \\
0 \longrightarrow a C \stackrel{\nu}{\longrightarrow} C \longrightarrow C / a C \longrightarrow 0
\end{aligned}
$$

where $\nu$ is the inclusion mapping and $\mu$ differs from $m_{a}$ only by the obvious contraction of the range. Since $a C_{a}=0$ and $a$ operates faithfully on $A$, we obtain from Lemma 3.2 that $\operatorname{Ext}\left(A, C_{a}\right)=0$, and so the relevant portions of the resulting cohomology sequences are as follows:

$$
0 \longrightarrow \operatorname{Ext}(A, C) \stackrel{\mu_{*}}{\longrightarrow} \operatorname{Ext}(A, a C) \longrightarrow 0
$$

$\operatorname{Hom}(A, C) \longrightarrow \operatorname{Hom}(A, C / a C) \longrightarrow \operatorname{Ext}(A, a C) \stackrel{\nu_{*}}{\longrightarrow} \operatorname{Ext}(A, C)$.

Since $m_{a}=\nu \mu$, we have that $m_{a *}=\nu_{*} \mu_{*}$, where $m_{a *}: \operatorname{Ext}(A, C) \rightarrow$ $\operatorname{Ext}(A, C)$ is defined by $m_{a *}(u)=a u$ for $u$ in $\operatorname{Ext}(A, C)$. Hence (a) holds if and only if $m_{a *}$ is a monomorphism. But this is true if and only if $\nu_{*}$ is a monomorphism, since $\mu_{*}$ is an isomorphism. But it is clear from the second exact sequence above that $\nu_{*}$ is a monomorphism if and only if (b) holds. The proof is hence complete. 
In the remainder of this section we shall set $\Pi=\Pi_{i=1}^{\infty} R^{(i)}$, where $R^{(i)} \approx R$.

THEOREM 3.4. Let $R$ be a Dedekind ring, and $a \neq 0$ be a nonunit in $R$. Set $C=\sum_{n=1}^{\infty} \oplus R / a^{n} R$. Let $A$ be a torsion-free $R$-module satisfying the following conditions:

(a) Every submodule of $A$ of finite rank is projective.

(b) a operates faithfully on $\operatorname{Ext}(A, C)$.

Then, if $f \in \operatorname{Hom}(I, A), f(I)$ has finite rank.

Proof. Assume that the statement is false for some $f \in \operatorname{Hom}(\Pi, A)$. Then $f(I)$ contains a submodule $F_{0}$ of countably infinite rank. Let $F=\left\{x \in A / a^{n} x \in F_{0}\right.$ for some $\left.n\right\}$. Then $F$ likewise has countably infinite rank. We may then apply condition (a) and a result of Nunke ([8], Lemma 8.3, p. 239) to obtain that $F$ is projective, and then a result of Kaplansky ([7], Theorem 2, p. 330) to conclude that $F$ is free. Let $x_{1}, x_{2}, \cdots$ be a basis of $F$. Then there exist nonnegative integers $\nu_{1}, \nu_{2}, \cdots$ such that $y_{n}=a^{\nu} x_{n}$ is in $F_{0}$.

Let $z_{n}$ generate the direct summand of $C$ isomorphic to $R / a^{n} R$, and let $\bar{z}_{n}$ be the image of $z_{n}$ under the natural mapping of $C$ onto $\bar{C}=C / a C$. Define an $R$-homomorphism $\theta_{1}: F \rightarrow \bar{C}$ by $\theta_{1}\left(x_{n}\right)=\bar{z}_{n+\nu_{n}}$. Observe that $\theta_{1}(a F)=0$, and so $\theta_{1}$ induces a homomorphism $\theta_{2}: F / a F \rightarrow \bar{C}$. Now, it follows easily from the construction of $F$ that the sequence $0 \rightarrow F / a F \rightarrow$ $A / a F \rightarrow A / F \rightarrow 0$ is exact, and $a$ operates faithfully on $A / F$. We may then apply Lemma 3.2 to conclude that this sequence splits. It is then clear that $\theta_{2}$ can be extended to a homomorphism $\theta: A \rightarrow \bar{C}$. We emphasize the fact that $\theta\left(x_{n}\right)=\bar{z}_{n+\nu_{n}}$.

Since a operates faithfully on $\operatorname{Ext}(A, C)$, we may now apply Lemma 3.3 to obtain $\varphi \in \operatorname{Hom}(A, C)$ such that the diagram--

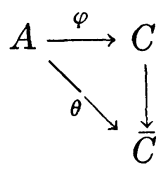

is commutative. Observe that, since $\theta\left(x_{n}\right)=\bar{z}_{n+\nu_{n}}, \varphi\left(x_{n}\right) \equiv z_{n+\nu_{n}}(\bmod a C)$. That is, the coefficient of $z_{n+\nu_{n}}$ in the expansion of $\varphi\left(x_{n}\right)$ is $1+a t_{n}$ for some $t_{n} \in R$. Since $y_{n}=a^{\nu} x_{n}$, the coefficient of $z_{n+\nu_{n}}$ in the expansion of $\varphi\left(y_{n}\right)$ is $a^{\nu n}+a^{\nu} n^{+1} t_{n}$.

Set $g=\varphi f$; then $g \in \operatorname{Hom}(\Pi, C)$, and so we may apply Proposition 2.4 to conclude that $c g(I)=0$ for some $c \neq 0$ in $R$. Since each $y_{n}$ is in $f(I I)$, and $z_{n}$ generates a direct summand of $C$ isomorphic to $R / a^{n} R$, it then follows from the preceding paragraph that $c\left(a^{\nu} n+a^{\nu_{n}+1} t_{n}\right)$ is in $a^{n+\nu_{n}} R$ for all $n$, in which case $c\left(1+a t_{n}\right)$ is in $a^{n} R$ for all $n$. Let $P$ 
be any prime ideal in $R$ containing $a$; then $1+a t_{n}$ is a unit modulo $P^{n}$ for all $n>0$, and so $c \in P^{n}$ for all $n$. Therefore $c=0$, a contradiction. This completes the proof of the theorem.

COROLlaRY 3.5. Let $R$ be a Dedekind ring (not a field,) and let $A$ be an $R$-module with the property that $\operatorname{Ext}(A, C)=0$ for any torsion module $C$. Then, if $f \in \operatorname{Hom}(\Pi, A), f(\Pi)$ is a projective module of finite rank.

Proof. We may apply a result of Nunke ([8], Theorem 8.4, p. 239) to obtain that $A$ is torsion-free and every submodule of $A$ of finite rank is projective. The corollary then follows immediately from Theorem 3.4 .

The following special case of Theorm 3.4 was first proved by Rotman ([10], Theorem 3, p. 250) under an additional hypothesis whitch was later removed by Nunke ([9], p. 275.)

CoRollary 3.6. Let $A$ be an abelian group such that $\operatorname{Ext}(A, C)=$ 0 for any torsion group $C$. Then $A$ is slender. ${ }^{3}$

Proof. We need only show that, for any $f \in \operatorname{Hom}(I, A), f(I I)$ is slender. By Corollary 3.5, $f(\Pi)$ is free of finite rank. But it is wellknown that a free abelian group is slender (see [5], Theorems 47.3 and 47.4, pp. 171-172.) The proof is hence complete.

\section{REFERENCES}

1. R. Baer, Die Torsionsuntergruppe Einer Abelschen Gruppe, Math. Annalen, 135 (1958), 219-234.

2. G. Baumslag and N. Blackburn, Direct summands of unrestricted direct sums of Abelian groups, Arkiv Der Mathematik, 10 (1959), 403-408.

3. S. Chase, Direct products of modules, Trans. Amer. Math. Soc., 97 (1960), 457-473.

4. - A remark on direct products of modules, Proc. Amer. Math. Soc., 13 (1962), 214-216.

5. L. Fuchs, Abelian Groups, Publishing House of the Hungarian Academy of Sciences, 1958.

6. I. Kaplansky, In finite Abelian Groups, University of Michigan Press, 1954.

7. - Modules over Dedekind rings and valuation rings, Trans. Amer. Math. Soc., 72 (1952), 327-340.

8. R. Nunke, Modules of extensions over Dedekind rings, Ill. Math. J., 3 (1959), 222-241.

9. - Slender groups, Bull. Amer. Math. Soc., 67 (1961), 274-275.

10. J. Rotman, On a problem of Baer and a problem of Whitehead in Abelian groups, Acta. Math. Sci. Hung., 12 (1961), 245-254.

PRINCETON UNIVERSITY

${ }^{3}$ For the definition of a slender Abelian group we refer the reader to [9]. 


\title{
PACIFIC JOURNAL OF MATHEMATICS
}

\author{
EDITORS
}

RalPh S. Phillips

Stanford University

Stanford, California

M. G. Arsove

University of Washington

Seattle 5 , Washington
A. L. Whiteman

University of Southern California Los Angeles 7, California

LOWell J. PAIGE

Unıversity of California

Los Angeles 24, California

\section{ASSOCIATE EDITORS}
E. F. BECKENBACH
D. DERRY
H. L. ROYDEN
E. G. STRAUS
T. M. CHERRY
M. OHTSUKA
E. SPANIER
F. WOLF

\section{SUPPORTING INSTITUTIONS}

\author{
UNIVERSITY OF BRITISH COLUMBIA \\ CALIFORNIA INSTITUTE OF TECHNOLOGY \\ UNIVERSITY OF CALIFORNIA \\ MONTANA STATE UNIVERSITY \\ UNIVERSITY OF NEVADA \\ NEW MEXICO STATE UNIVERSITY \\ OREGON STATE UNIVERSITY \\ UNIVERSITY OF OREGON \\ OSAKA UNIVERSITY \\ UNIVERSITY OF SOUTHERN CALIFORNIA
}

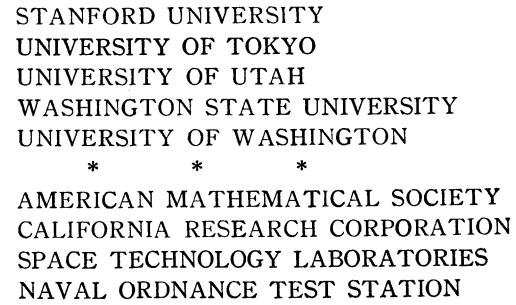

Mathematical papers intended for publication in the Pacific Journal of Mathematıcs should be typewritten (double spaced), and the author should keep a complete copy. Manuscripts may be sent to any one of the four editors. All other communications to the editors should be addressed to the managing editor, L. J. Paige at the University of California, Los Angeles 24, California.

50 reprints per author of each article are furnıshed free of charge; additional copies may be obtained at cost in multiples of 50 .

The Pacific Journal of Mathematics is published quarterly, in March, June, September, and December. Effective with Volume 13 the price per volume (4 numbers) is $\$ 18.00$; single issues, $\$ 5.00$. Special price for current issues to individual faculty members of supporting institutions and to individual members of the American Mathematical Society: $\$ 8.00$ per volume; single issues $\$ 2.50$. Back numbers are available.

Subscriptions, orders for back numbers, and changes of address should be sent to Pacific Journal of Mathematics, 103 Highland Boulevard, Berkeley 8, California.

Printed at Kokusai Bunken Insatsusha (International Academic Printing Co., Ltd.), No. 6 , 2-chome, Fujimi-cho, Chiyoda-ku, Tokyo, Japan.

PUBLISHED BY PACIFIC JOURNAL OF MATHEMATICS, A NON-PROFIT CORPORATION

The Supporting Institutions listed above contribute to the cost of publication of this Journal, but they are not owners or publishers and have no responsibility for its content or policies. 


\section{Pacific Journal of Mathematics}

\section{Vol. 12, No. $3 \quad$ March, 1962}

Alfred Aeppli, Some exact sequences in cohomology theory for Kähler

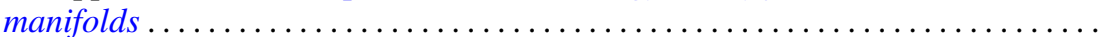

Paul Richard Beesack, On the Green's function of an N-point boundary value

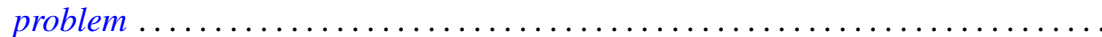

James Robert Boen, On p-automorphic p-groups....

James Robert Boen, Oscar S. Rothaus and John Griggs Thompson, Further results

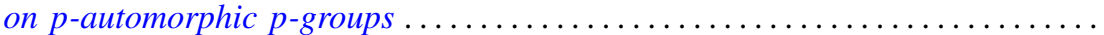

James Henry Bramble and Lawrence Edward Payne, Bounds in the Neumann problem for second order uniformly elliptic operators ..................

Chen Chung Chang and H. Jerome (Howard) Keisler, Applications of ultraproducts of pairs of cardinals to the theory of models .........................

Stephen Urban Chase, On direct sums and products of modules ................

Paul Civin, Annihilators in the second conjugate algebra of a group algebra .......

J. H. Curtiss, Polynomial interpolation in points equidistributed on the unit

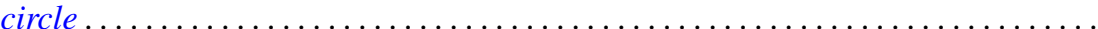

Marion K. Fort, Jr., Homogeneity of infinite products of manifolds with

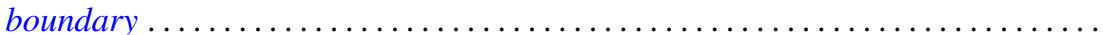

James G. Glimm, Families of induced representations . . . . . . . . . . . . . . .

Daniel E. Gorenstein, Reuben Sandler and William H. Mills, On almost-commuting

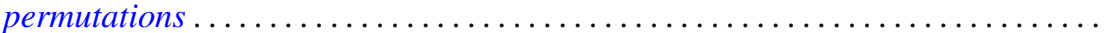

Vincent C. Harris and M. V. Subba Rao, Congruence properties of $\sigma_{r}(N) \ldots \ldots \ldots$

Harry Hochstadt, Fourier series with linearly dependent coefficients . . . . . . . . . . 925

Kenneth Myron Hoffman and John Wermer, A characterization of $C(X)$. .

Robert Weldon Hunt, The behavior of solutions of ordinary, self-adjoint differential equations of arbitrary even order...

Edward Takashi Kobayashi, A remark on the Nijenhuis tensor

David London, On the zeros of the solutions of $w^{\prime \prime}(z)+p(z) w(z)=0$

Gerald R. Mac Lane and Frank Beall Ryan, On the radial limits of Blaschke products...

T. M. MacRobert, Evaluation of an E-function when three of its upper parameters

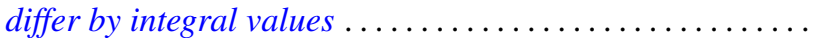

Robert W. McKelvey, The spectra of minimal self-adjoint extensions of a symmetric operator

Adegoke Olubummo, Operators of finite rank in a reflexive Banach space. .

David Alexander Pope, On the approximation of function spaces in the calculus of variations

Bernard W. Roos and Ward C. Sangren, Three spectral theorems for a pair of singular first-order differential equations...............

Arthur Argyle Sagle, Simple Malcev algebras over fields of characteristic zero .

S. C. Tang, Some theorems on the ratio of empirical distribution to the theoretical distribution

Robert Charles Thompson, Normal matrices and the normal basis in abelian number fields.

Howard Gregory Tucker, Absolute continuity of infinitely divisible distributions ... 